\title{
Methyl-CpG binding domain protein 3: a new diagnostic marker and potential therapeutic target of melanoma
}

\author{
Takayuki Ishibashi $^{1}$, Ikko Kajihara ${ }^{1}$, Satoru Mizuhashi ${ }^{1}$, Haruka Kuriyama ${ }^{1}$, \\ Toshihiro Kimura ${ }^{1}$, Hisashi Kanemaru ${ }^{1}$, Katsunari Makino ${ }^{1}$, Azusa Miyashita ${ }^{1}$, \\ Jun Aoi ${ }^{1}$, Takamitsu Makino ${ }^{1}$, Satoshi Fukushima ${ }^{1, *}$, Kanako Kita ${ }^{2, *}$, Hironobu Ihn ${ }^{1}$ \\ ${ }^{1}$ Department of Dermatology and Plastic Surgery, Graduate School of Medical Sciences, Kumamoto University, Kumamoto, Japan; \\ ${ }^{2}$ Department of Molecular Pathology, Graduate School of Medical Sciences, Kumamoto University, Kumamoto, Japan.
}

SUMMARY Methyl-CpG binding domain protein 3 (MBD3) belongs to the methyl-CpG binding protein family. MBD3 facilitates the initiation of neural stem cell reprogramming. Melanoma originates in melanocytes derived from neural crest stem cells; therefore, we investigated the role of MBD3 in melanoma. MBD3 was overexpressed in melanoma compared with pigmented nevi. MBD3 knockdown had no effect on the proliferation of melanoma cells (A375 and A2058 cells). Contrarily, it significantly reduced the migration and invasion of A375 cells, but had no significant effect on A2058 cells. Furthermore, MBD3 knockdown reduced N-cadherin protein levels and matrix metalloproteinase-2 (MMP-2) activity in A375 cells, but had no significant effect on A2058 cells. Based on these results, the MBD3 expression level may be a useful biomarker for the diagnosis of melanoma. Thus, MBD3 has potential as a novel therapeutic target for some melanoma patients.

Keywords $\quad$ MBD3, melanoma, N-cadherin, MMP-2

\section{Introduction}

Although immune checkpoint inhibitors (ICIs) improve the prognosis of patients with advanced melanoma, the response rate to ICIs is approximately $30-40 \%$ (1). In addition, only approximately $30 \%$ of Japanese melanoma patients have a BRAF mutation, which is required for treatment with BRAF inhibitors (1). As such, the currently available therapies are not suitable for all melanoma patients. Therefore, novel therapeutic molecular targets for advanced melanoma need to be identified. Furthermore, it is not always possible to make a differential diagnosis between a nevus and a melanoma. This is due to the fact that the representative melanoma markers, including melanoma antigen recognized by $\mathrm{T}$ cells 1 (MART-1) and gp100, are also present in pigmented nevi. Therefore, they are not useful for differential diagnoses.

Methyl-CpG binding domain protein 3 (MBD3) is approximately $35 \mathrm{kDa}$ and belongs to the methyl-CpGbinding protein family. MBD3 acts as a transcriptional repressor through its interaction with nucleosome remodeling deacetylase (NuRD) (2). MBD3 is essential for the formation and stability of the NuRD complex (3). It is contained within this complex, where it binds to hydroxymethylated DNA (4). DNA hydroxymethylation is an epigenetic mechanism that modifies the C-5 position of cytosine by adding a hydroxymethyl group, resulting in the regulation of gene expression levels $(5,6)$. MBD3 binds to hydroxymethylated DNA and suppresses gene expression (4). MBD3 protein has been previously detected in neural stem cells using two-dimensional fluorescence differential gel electrophoresis targeting nuclear phosphorylated proteins after stimulation with fibroblast growth factor 2 (7). MBD3 is involved in the regulation of neural stem cell reprogramming and differentiation (8). Moreover, melanoma originates from melanocytes derived from neural crest cells (9).

Although the expression levels of MBD3 are high in several cancers, there is a divergence in terms of its function according to the type of cancer. MBD3 suppresses tumor growth in lung cancer (10) and pancreatic cancer (11) but promotes tumor growth in breast cancer (12). However, the role of MBD3 in melanoma has not yet been clarified. Therefore, we investigated the role of MBD3 in melanoma and whether the inhibition of MBD3 has an antitumor effect.

\section{Material and Methods}

2.1. Clinical assessment and patient samples 
In accordance with the Declaration of Helsinki, institutional review board approval and written informed consent was obtained from patients before their enrollment in this study. Skin samples were collected from 20 patients with melanoma and 19 patients with pigmented nevi.

\subsection{Cell culture}

Human melanoma cell lines were obtained from the Cell Resource Center for Biomedical Research Institute of Development, Aging and Cancer, Tohoku University (Sendai, Miyagi, Japan) or the American Type Culture Collection (Manassas, VA, USA). Normal human epidermal melanocytes (NHEM) were purchased from Lonza (Basel, Switzerland). Human melanoma cell lines were maintained in Dulbecco's modified Eagle medium, supplemented with $20 \%$ fetal bovine serum (FBS) under 5\% $\mathrm{CO}_{2}$ and 95\% air. NHEM in CSF-4HM-500D culture medium, supplemented with human melanocyte growth supplements, were maintained under $5 \% \mathrm{CO}_{2}$ and $95 \%$ air.

\subsection{Immunofluorescent staining}

Immunofluorescent staining of MBD3 and Melan-A proteins in patient tissues and cultured cell lines was performed using an anti-MBD3 antibody at a dilution of 1:100 (ab157464; Abcam, Cambridge, UK) or an antiMelan-A antibody at 1:100 (mouse monoclonal) (ab731; Abcam), respectively. Slides were counterstained with Fluoroshield mounting medium with 4',6-diamidino-2phenylindole (DAPI) (ab104139; Abcam) and images were captured using fluorescence microscopy (BZ-X 710; Keyence, Osaka, Japan). The intensity of staining was classified as follows: (-), same or weaker than the adjacent epidermis; $(+)$, stronger than the adjacent epidermis; $(++)$, much stronger than the adjacent epidermis.

\subsection{Western blotting}

Equal amounts of proteins $(10 \mu \mathrm{g})$ were separated using SDS polyacrylamide gels and were electrotransferred to polyvinylidene fluoride (PVDF) membranes (Bio-Rad Laboratories, Hercules, CA, USA). The membranes were immunoblotted overnight at $4^{\circ} \mathrm{C}$ with primary antibodies, followed by their respective secondary antibodies, anti-MBD3 (1:1,000; Abcam), anti-Ncadherin (1:1,000; Abcam), and anti- $\beta$-actin $(1: 2,000$; Cell Signaling Technology, Beverly, MA, USA).

\subsection{Gene silencing using small interfering RNA}

An MBD3-specific small interfering RNA (siRNA) and a scrambled control siRNA were purchased from Dharmacon (Lafayette, CO, USA). The target sequences of the MBD3-specific siRNA are the following four sequences: CCUGAACGCCUUCGACAUU, UGAGCA AGAUGAACAAGAG, UCAAGCAGCCGGUGACCA A, CCAACCAGGUCAAGGGCAA. Human melanoma cell lines were transfected using Lipofectamine RNAiMAX transfection reagent (Invitrogen Corporation, Carlsbad, CA, USA) for $6 \mathrm{~h}$ following manufacturer's instructions. The final concentration of MBD3-specific siRNA and scrambled control siRNA were $100 \mathrm{nM}$.

\subsection{Cell proliferation assay}

A375 (low metastatic melanoma cell line) and A2058 (high metastatic melanoma cell line) (13) cells were seeded at $5.0 \times 10^{4}$ cells/well in 6 -well plates and transfected with either an $M B D 3$-specific siRNA or a scrambled control siRNA using Lipofectamine RNAiMAX. After incubating for 48 hours, the transfected cells were stained with Trypan blue and counted under a light microscope. Each experiment was performed in triplicate.

\subsection{Migration and invasion assays}

Migration and invasion assays were performed to evaluate the migrative and invasive ability of A375 and A2058 cells transfected with either an MBD3specific siRNA or a scrambled control siRNA using Lipofectamine RNAiMAX. For migration assay, a 24well plate containing Permeable Support with $8.0 \mu \mathrm{m}$ Transparent PET Membrane (Corning Inc., Corning, NY, USA), was prepared and for invasion assay, a 24-well plate containing $8 \mu \mathrm{m}$ pore size transwell inserts pre-coated with Matrigel (Corning Inc.) was prepared. After serum starved incubation with serumfree Dulbecco's Modified Eagle Medium (DMEM) in $5 \% \mathrm{CO}_{2}$ atmosphere at $37^{\circ} \mathrm{C}$ for $24 \mathrm{~h}$, the cells were seeded into the upper chamber of the insert at $5 \times 10^{4} /$ well in $500 \mu \mathrm{L}$ serum-free DMEM. The lower chamber was filled with $750 \mu \mathrm{L}$ DMEM supplemented with $20 \%$ fetal bovine serum as a chemoattractant. After incubating the cells at $37^{\circ} \mathrm{C}$ for $48 \mathrm{~h}$, the cells on the upper chamber of the insert were removed with a cotton swab. Subsequently, the cells on the bottom of the insert were fixed with paraformaldehyde for 15 min. Using an inverted microscope, the migrated and invaded cells were counted in five different fields at $200 \times$ magnification.

\subsection{MMP-2 activity assay}

Supernatants were collected from melanoma cells (A375 and A2058 cells) and cultured in 6-well plates for 48 hours. MMP-2 activity was measured using a commercially available assay (QuickZyme Biosciences, Leiden, Netherlands), according to the manufacturer's protocol. 


\subsection{Statistical analysis}

Data are presented as bar graphs with the mean \pm standard deviation (SD) of at least three independent experiments. Statistical analyses were performed using the Mann-Whitney $U$-test to compare medians. The immunofluorescent staining results were analyzed using the Chi-squared test. A $p$-value $<0.05$ was considered to be statistically significant.

\section{Results}

\subsection{MBD3 was overexpressed in melanoma}

Western blotting was performed to examine the MBD3 expression levels in melanoma in vitro. The MBD3 levels were higher in melanoma cell lines than in NHEM (Figure 1a). Immunofluorescent staining showed results similar to western blotting (Figure 1b). In addition, we measured the expression levels of MBD3 in melanoma tissues by immunofluorescence. A representative MBD3

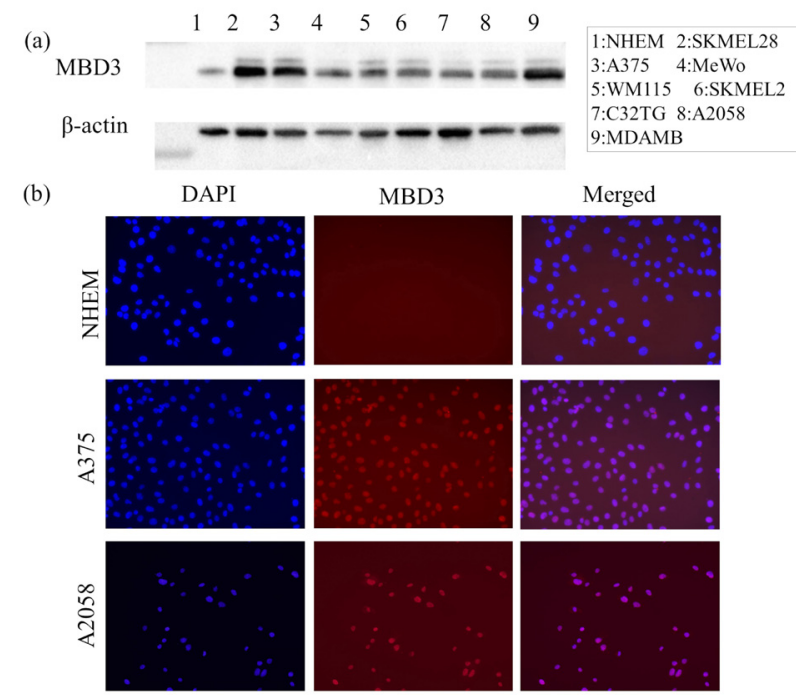

Figure 1. (a) Expression levels of methyl-CpG binding domain protein 3 (MBD3) protein in melanoma and normal human epidermal melanocyte (NHEM) cell lines using immunoblotting. (b) Immunofluorescent staining. Expression of MBD3 protein in A375, A2058, and NHEM cell lines. MART-1 is shown in green. MBD3 is shown in red. DNA is stained blue (DAPI).

Table 1. Results of the immunofluorescent analysis of MBD3

\begin{tabular}{lcccc}
\hline Items & $n$ & - & + & ++ \\
\hline Pigmented nevus & 19 & 16 & 2 & 1 \\
Melanoma & 20 & 3 & 1 & 16
\end{tabular}

The tissue samples were classified as negative (-), slightly positive (+), or strongly positive (++) based on MBD3 immunoreactivity. MBD3 staining was significantly more intense in melanoma samples than in pigmented nevi. immunofluorescence experiment is shown in Figure $2 \mathrm{a}$. The tissue samples were classified as negative, slightly positive, or strongly positive based on MBD3 immunoreactivity (Figure 2b). MBD3 staining was significantly more intense in melanoma samples than in pigmented nevi (Table 1). Moreover, when the patients were evaluated by the staining results of MBD3, neither lymph node metastasis nor organ metastasis was observed in all three cases of melanoma negative for MBD3 (Table 2).

3.2. An MBD3-specific small interfering RNA inhibited the migration and invasion of A375 cells

We investigated the effect of an MBD3-specific siRNA in melanoma cell lines (A375 and A2058 cells) to determine the role of MBD3 in the pathogenesis of melanoma. The expression level of MBD3 was downregulated by the MBD3-specific siRNA, as shown in Figure 3a. MBD3 knockdown did not affect the proliferation of neither A375 nor A2058 cells (Figure

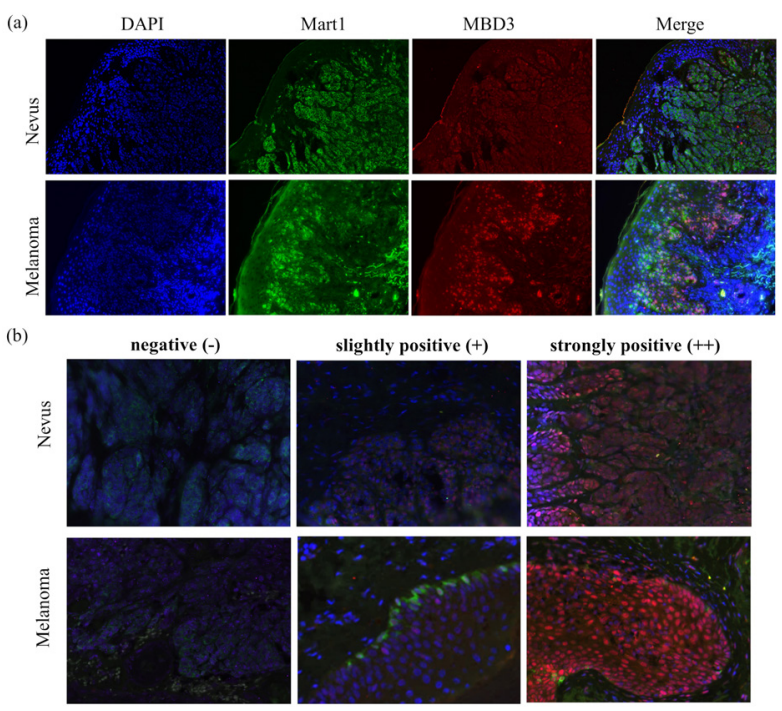

Figure 2. Immunofluorescent staining in melanoma and pigmented nevi. (a) Representative images of immunofluorescent staining of nuclei (DAPI, blue), MART-1 protein (green), and MBD3 protein (red) in melanoma and nevus tissue samples. (400× magnification). (b) Representative images of semiquantitative scoring of immunofluorescent staining. The intensity of staining was classified as follows: $(-)$, same or weaker than the adjacent epidermis; $(+)$, stronger than the adjacent epidermis; or $(++)$, much stronger than the adjacent epidermis.

Table 2. Correlation between MBD3 immunofluorescent staining and clinical features of melanoma patients

\begin{tabular}{lccc}
\hline Items & $\begin{array}{c}\text { Negative } \\
(n=3)\end{array}$ & $\begin{array}{c}\text { Positive } \\
(n=17)\end{array}$ & $p$-value \\
\hline Sex (Male:Female) & $2: 1$ & $8: 9$ & 1 \\
Age (years), mean \pm SD & $74.9 \pm 11.7$ & $63.3 \pm 21.7$ & 0.175 \\
Lymph node metastasis (No:Yes) & $3: 0$ & $9: 8$ & 0.242 \\
Organ metastasis (No:Yes) & $3: 0$ & $15: 2$ & 1 \\
\hline
\end{tabular}


(a)
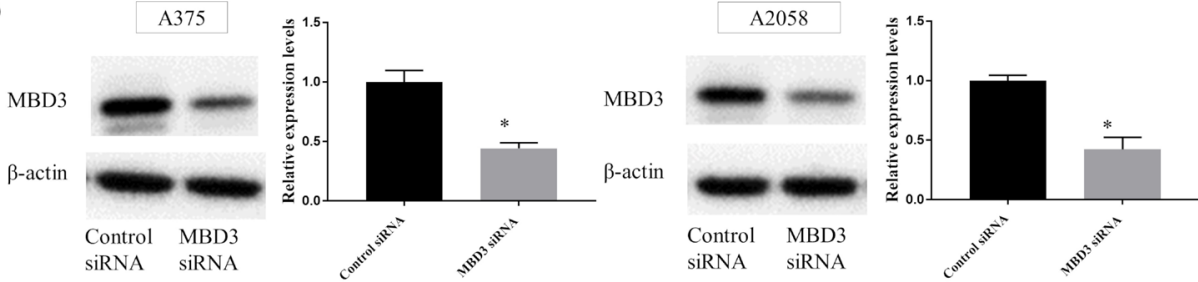

(b)
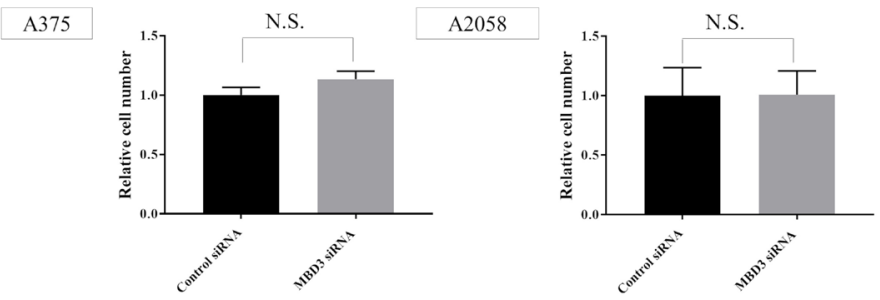

(c)
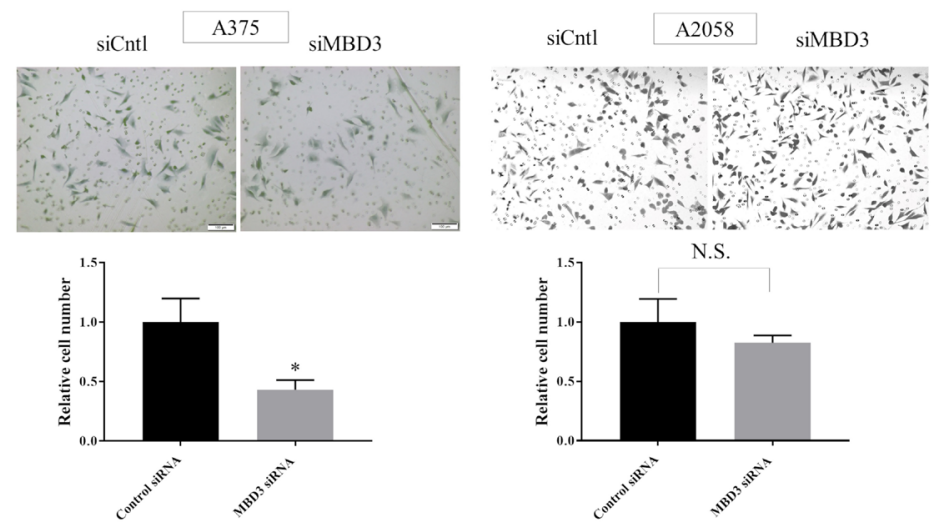

(d)
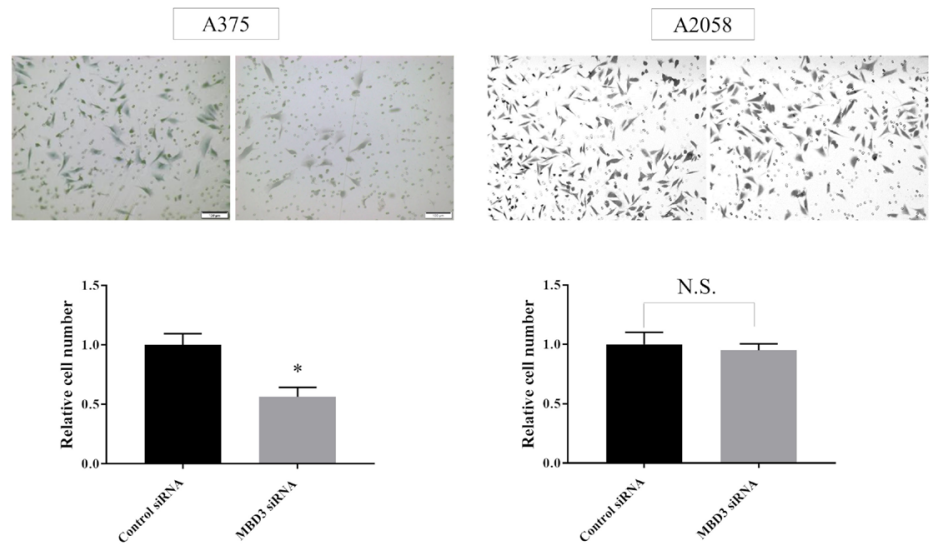

Figure 3. Knockdown of MBD3 affected cell growth, migration, and invasion in melanoma. (a) Down-regulation of MBD3 expression by small interfering RNA (siRNA). A375 and A2058 cells were transfected with a control or MBD3-specific siRNA. After treatment for 48 hours, we evaluated cell proliferation, migration, and invasion. Data represent the mean \pm SD from three independent experiments. (b) The number of melanoma cells was counted using a particle counter. (c) Cell migration was evaluated using transwell inserts without a Matrigel coating. (d) Cell invasion was evaluated using transwell inserts coated with Matrigel. Diff-Quick staining of melanoma cells treated with a control or MBD3specific siRNA. Magnification, $400 \times$. Data are expressed as the mean \pm SD of three independent experiments. $p<0.05$ versus controls.

3b). We also evaluated the effect of the MBD3-specific siRNA on the migration and invasion of melanoma cells. Migration/invasion assays showed that MBD3 silencing significantly inhibited the migration and invasion of A375 cells, but had no statistically significant effect on the migration or invasion of A2058 cells (Figures 3c and 3d).

\subsection{MBD3 knockdown suppressed N-cadherin} expression and MMP-2 activity in A375 cells
$\mathrm{N}$-cadherin promotes the migration of melanocytes and is involved in the migratory ability of melanoma (14). MMP-2 has the ability to degrade type IV collagen and is associated with the migration and invasion of cancer (15). To clarify the mechanism of migration and invasion related to MBD3, we examined whether MBD3 knockdown affected $\mathrm{N}$-cadherin expression levels and MMP-2 activity in melanoma cell lines. As shown in Figure 4a-b, MBD3 knockdown significantly suppressed N-cadherin expression and MMP-2 activity 
(a)
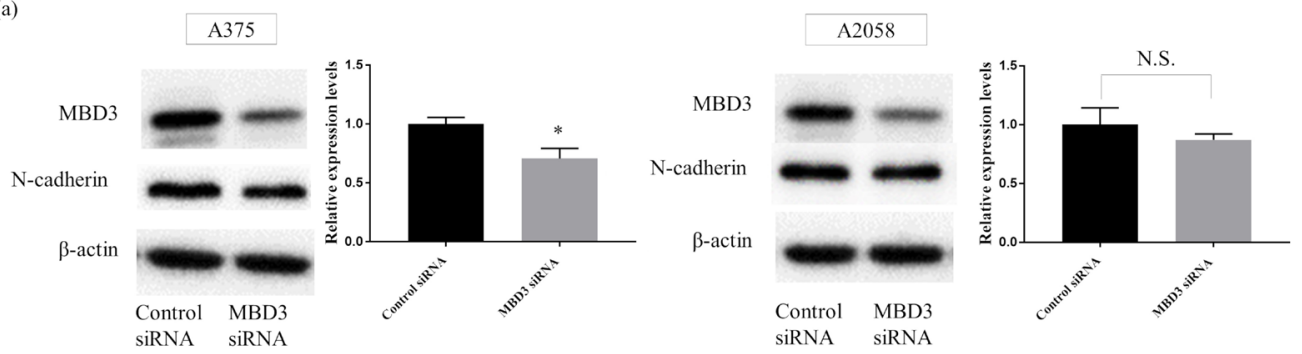

(b)

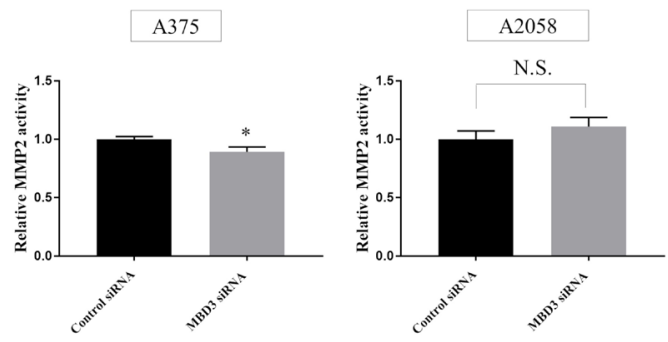

Figure 4. Knockdown of MBD3 down-regulated N-cadherin and inhibited MMP-2 activity in A375 cells, but not A2058 cells. A375 and A2058 cells were transfected with a control or MBD3-specific siRNA. All results were analyzed after 48 hours of treatment. The levels of N-cadherin expression (a, western blotting) and MMP-2 activity (b) in A375 and A2058 cells. Data are expressed as the mean \pm SD of three independent experiments. ${ }^{*} p<0.05$ versus controls. N.S., not significant.

in A375 cells, but had no effect on these parameters in A2058 cells.

\section{Discussion}

In this study, we revealed two major findings. Firstly, MBD3 was found to be strongly expressed in the cultured melanoma cells and tissues of melanoma patients. In addition, as shown in Figure $2 \mathrm{~b}$ and Table 1, the MBD3 levels were significantly higher in melanoma compared with pigmented nevi. These results demonstrated that MBD3 may be useful for the differential diagnosis of melanoma and pigmented nevus.

Secondly, the role of MBD3 in the progression of melanoma was found to vary depending on the cell line. MBD3 knockdown did not affect the proliferative ability of A375 or A2058 cells. However, MBD3 knockdown significantly reduced the migration and invasion of A375 cells, but had no significant effect on A2058 cells. Furthermore, the knockdown of MBD3 reduced the $\mathrm{N}$-cadherin protein levels and MMP2 activity in A375 cells, but had no significant effect on A2058 cells. These findings suggested that MBD3 may promote migration and invasion by regulating $\mathrm{N}$-cadherin and MMP-2 in A375 cells. The reason of the finding that the migration and invasion of A2058 cells were not affected by an MBD3-specific siRNA, was suggested to depend on the presence of PTEN. A2058 cells have been established from metastatic lymph node and reported to be PTEN-deficient, while A375 cells have wild-type PTEN $(16,17)$. PTEN is a negative regulator of $\mathrm{PI} 3 \mathrm{~K}$, and the inactivation of PTEN can promote the metastatic progression of melanoma (18). The progression of melanoma has characteristic features of epithelial to mesenchymal transition (EMT), including the disruption of the adherent junctions caused by the upregulation of N-cadherin. The PI3K PTEN pathway transcriptionally regulates this cadherin regulation (19). It has been reported that PTEN was repressed through the epigenetic repressor NuRD complex (20). MBD3 is essential for the formation and stability of the NuRD complex. The knockdown of MBD3 may only suppress EMT in tumor cells with PTEN. Therefore, we suggest that the migration and invasion of PTEN-deficient A2058 cells were not affected by an MBD3-specific siRNA. However, this should be confirmed using a greater number of different cell lines in future studies.

In conclusion, although the differential diagnosis of pigmented nevus and melanoma is sometimes difficult, the assessment of MBD3 protein expression levels may solve this problem. Although heterogeneity was observed depending on the type of cell line, MBD3 has potential for use as a therapeutic target for the treatment of advanced melanoma.

\section{Acknowledgements}

This work was supported by a JSPS Grant (grant numbers $15 \mathrm{~K} 09772$ and 18K08301).

\section{References}

1. Nakamura Y, Asai J, Igaki H, et al. Japanese Dermatological Association Guidelines: Outlines of guidelines for cutaneous melanoma 2019. J Dermatol. 2020; 47:89-103.

2. Dos Santos RL, Tosti L, Radzisheuskaya A, Caballero IM, 
Kaji K, Hendrich B, Silva JCR. MBD3/NuRD Facilitates Induction of Pluripotency in a Context-Dependent Manner. Cell Stem Cell. 2014; 15:102-110.

3. Wade PA, Gegonne A, Jones PL, Ballestar E, Aubry F, Wolffe AP. Mi-2 complex couples DNA methylation to chromatin remodelling and histone deacetylation. Nat Genet. 1999; 23:62-66.

4. Yildirim O, Li R, Hung JH, Chen PB, Dong X, Ee LS, Weng Z, Rando OJ, Fazzio TG. Mbd3/NURD complex regulates expression of 5-hydroxymethylcytosine marked genes in embryonic stem cells. Cell. 2011; 147:14981510.

5. Wu H, Zhang Y. Tet1 and 5-hydroxymethylation: A genome-wide view in mouse embryonic stem cells. Vol. 10, Cell Cycle. Taylor and Francis Inc., 2011; pp. 24282436.

6. Mendonca A, Chang EH, Liu W, Yuan C. Hydroxymethylation of DNA influences nucleosomal conformation and stability in vitro. Biochim Biophys Acta. 2014; 1839:1323-1329.

7. Niimori-Kita K, Tamamaki N, Koizumi D, Niimori D. Matrin-3 is essential for fibroblast growth factor 2-dependent maintenance of neural stem cells. Sci Rep. 2018; 8:13412.

8. Kaji K, Caballero IM, MacLeod R, Nichols J, Wilson VA, Hendrich B. The NuRD component Mbd3 is required for pluripotency of embryonic stem cells. Nat Cell Biol. 2006; 8:285-292.

9. Kohler C, Nittner D, Rambow F, Radaelli E, Stanchi F, Vandamme N, Baggiolini A, Sommer L, Berx G, van den Oord JJ, Gerhardt H, Blanpain C, Marine JC. Mouse cutaneous melanoma induced by mutant B-Raf arises from expansion and dedifferentiation of mature pigmented melanocytes. Cell Stem Cell. 2017; 21:679-693.e6.

10. Noh EJ, Jang ER, Jeong G, Lee YM, Min CK, Lee JS. Methyl CpG-binding domain protein 3 mediates cancerselective cytotoxicity by histone deacetylase inhibitors via differential transcriptional reprogramming in lung cancer cells. Cancer Res. 2005; 65:11400-11410.

11. Xu M, He J, Li J, Feng W, Zhou H, Wei H, Zhou M, Lu Y, Zeng J, Peng W, Du F, Gong A. Methyl-CpG-binding domain 3 inhibits epithelial-mesenchymal transition in pancreatic cancer cells via TGF-beta/Smad signalling. $\mathrm{Br}$ J Cancer. 2017; 116:91-99.

12. Cui J, Duan B, Zhao X, Chen Y, Sun S, Deng W, Zhang Y, Du J, Chen Y, Gu L. MBD3 mediates epigenetic regulation on EPAS1 promoter in cancer. Tumor Biol. 2016; 37:13455-13467.
13. Kim HY, Lee H, Kim SH, Jin H, Bae J, Choi HK. Discovery of potential biomarkers in human melanoma cells with different metastatic potential by metabolic and lipidomic profiling. Sci Rep. 2017; 7:8864.

14. Li G, Satyamoorthy K, Herlyn M. N-cadherin-mediated intercellular interactions promote survival and migration of melanoma cells. Cancer Res. 2001; 61:3819-3825.

15. Pereira AMM, Strasberg-Rieber M, Rieber M. Invasionassociated MMP-2 and MMP-9 are up-regulated intracellularly in concert with apoptosis linked to melanoma cell detachment. Clin Exp Metastasis. 2005; 22:285-295.

16. Pap M, Bátor J, Szeberényi J. Sensitivity of human malignant melanoma cell lines to Newcastle disease virus. Anticancer Res. 2015; 35:5401-5406.

17. Chatterjee N, Pazarentzos E, Mayekar MK, et al. Synthetic Essentiality of Metabolic Regulator PDHK1 in PTEN-Deficient Cells and Cancers. Cell Rep. 2019; 28:2317-2330.e8.

18. Kim M. Cooperative interactions of PTEN deficiency and RAS activation in melanoma metastasis. Small GTPases. 2010; $1: 161$

19. Hao L, Ha JR, Kuzel P, Garcia E, Persad S. Cadherin switch from $\mathrm{E}$ - to $\mathrm{N}$-cadherin in melanoma progression is regulated by the PI3K/PTEN pathway through Twist and Snail. Br J Dermatol. 2012; 166:1184-1197.

20. Lu J, Jeong HW, Kong N, Yang Y, Carroll J, Luo HR, Silberstein LE, Yupoma, Chai L. Stem cell factor SALL4 represses the transcriptions of PTEN and SALL1 through an epigenetic repressor complex. PLoS One. 2009; 4:e5577.

Received February 18, 2020; Revised August 29, 2020; Accepted September 7, 2020.

*Address correspondence to:

Satoshi Fukushima, Department of Dermatology and Plastic Surgery, Faculty of Life Sciences, Kumamoto University, 1-1-1 Honjo, Kumamoto, Japan.

E-mail: satoshi.fukushima.tb@gmail.com

Kanako Kita, Department of Comprehensive Molecular Medicine, Faculty of Life Sciences, Kumamoto University, 1-1-1 Honjo, Kumamoto, Japan.

E-mail: kitakana@kumamoto-u.ac.jp

Released online in J-STAGE as advance publication September 18, 2020. 